RECEIVED 2015 JANNUARY 29; ACCEPTED 2015 MAY 4; PUBLISHED 2015 MM DD

Preprint typeset using LTEX style emulateapj v. 08/22/09

\title{
A SYSTEMATIC STUDY OF CARBON-OXYGEN WHITE DWARF MERGERS: MASS COMBINATIONS FOR TYPE Ia SUPERNOVAE
}

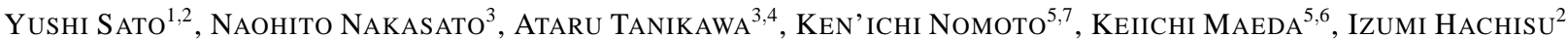 \\ ${ }^{1}$ Department of Astronomy, Graduate School of Science, The University of Tokyo, 7-3-1 Hongo, Bunkyo-ku, Tokyo 113-0033, Japan \\ ${ }^{2}$ Department of Earth Science and Astronomy, College of Arts and Sciences, The University of Tokyo, 3-8-1 Komaba, Meguro-ku, Tokyo 153-8902, Japan ; \\ sato@ea.c.u-tokyo.ac.jp \\ ${ }^{3}$ Department of Computer Science and Engineering, University of Aizu, Tsuruga Ikki-machi Aizu-Wakamatsu, Fukushima 965-8580, Japan \\ ${ }^{4}$ RIKEN Advanced Institute for Computational Science, 7-1-26, Minatojima-minami-machi, Chuo-ku, Kobe, Hyogo 650-0047, Japan \\ ${ }^{5}$ Kavli Institute for the Physics and Mathematics of the Universe (WPI), The University of Tokyo, 5-1-5 Kashiwanoha, Kashiwa, Chiba 277-8583, Japan \\ ${ }^{6}$ Department of Astronomy, Kyoto University, Kitashirakawa-Oiwake-cho, Sakyo-ku, Kyoto 606-8502, Japan \\ Received 2015 Jannuary 29; accepted 2015 May 4; published 2015 MM DD
}

\begin{abstract}
Mergers of two carbon-oxygen (CO) WDs have been considered as progenitors of Type Ia supernovae (SNe Ia). Based on smoothed particle hydrodynamics (SPH) simulations, previous studies claimed that mergers of CO WDs lead to an SN Ia explosion either in the dynamical merger phase or stationary rotating merger remnant phase. However, the mass range of CO WDs that lead to an SN Ia has not been clearly identified yet. In the present work, we perform systematic SPH merger simulations for the WD masses ranging from $0.5 M_{\odot}$ to $1.1 M_{\odot}$ with higher resolutions than the previous systematic surveys and examine whether or not carbon burning occurs dynamically or quiescently in each phase. We further study the possibility of SN Ia explosion and estimate the mass range of CO WDs that lead to an SN Ia. We found that when the both WDs are massive, i.e., in the mass range of $0.9 M_{\odot} \leq M_{1,2} \leq 1.1 M_{\odot}$, they can explode as an SN Ia in the merger phase. On the other hand, when the more massive WD is in the range of $0.7 M_{\odot} \leq M_{1} \leq 0.9 M_{\odot}$ and the total mass exceeds $1.38 M_{\odot}$, they can finally explode in the stationary rotating merger remnant phase. We estimate the contribution of CO WD mergers to the entire SN Ia rate in our galaxy to be of $\lesssim 9 \%$. So, it might be difficult to explain all galactic SNe Ia by CO WD mergers.

Subject headings: binaries: close — galaxies: evolution — supernovae: general — white dwarfs — hydrodynamics
\end{abstract}

\section{INTRODUCTION}

$\mathrm{SNe}$ Ia play the important roles in the determination of cosmological parameters as luminous standard candles (e.g., Riess et al. 1998; Perlmutter et al. 1999) and in the chemical evolution of galaxies as major sources of iron group elements (e.g., Kobayashi et al. 1998). However, their progenitors are still not identified yet (e.g., Maoz et al. 2014). They are considered as a thermonuclear explosion of a $\mathrm{CO}$ WDs in a binary system, in which the WD accretes mass from its companion and the WD mass approaches the Chandrasekhar mass $\left(\mathrm{M}_{\mathrm{Ch}} \sim 1.4 M_{\odot}\right)$. But, it is still controversial whether its companion is a non-degenerate star, i.e., single degenerate (SD) model, or a degenerate star, i.e., double degenerate (DD) model. In the SD model, a CO WD accretes hydrogen/helium-rich gas from the companion and increases its mass upto $M_{\mathrm{Ch}}$. Finally, carbon burning starts at the center of the CO WD and explodes as an SN Ia (Whelan \& Iben 1973; Nomoto 1982; Hachisu et al. 1996, 1999a,b). On the other hand, in the DD model, both the components are CO WDs. Because binaries lose their orbital angular momentum by emitting gravitational waves, they will eventually merge. If their total mass exceeds $M_{\mathrm{Ch}}$, the binary finally explodes as an SN Ia (Iben \& Tutukov 1984; Webbink 1984).

Some clues on the progenitors of SNe Ia were found in recent observations. In particular, neither surviving companions nor signatures of them were detected in some SNe Ia (e.g., Schaefer \& Pagnotta 2012). This fact supports the DD system, although no detection of companions can also be ex-

\footnotetext{
${ }^{7}$ Hamamatsu Professor
}

plained by the SD model (Di stefano et al. 2011; Justhum 2011; Hachisu et al. 2012). In some SNe Ia, signatures of circumstellar matter (CSM) were detected (e.g., Patat et al. 2007; Blondin et al. 2009; Simon et al. 2009; Sternberg et al. 2011). This supports the SD model (e.g., Maoz et al. 2014, for a recent review), although this can be explained by some DD models (Raskin \& Kasen 2013; Shen et al. 2013; Soker et al. 2013). Observations do still not clarify which model is the main progenitors of SNe Ia, i.e., SD or DD (or other type) system.

The DD model has a theoretical difficulty as a progenitor model. Some theoretical studies indicated that CO WD mergers can not become an SN Ia, but collapse to a neutron star (e.g., Saio \& Nomoto 1985, 2004; Nomoto \& Kondo 1991). These studies calculated only the evolution of merger remnants after DD systems merged. Their calculations were onedimensional (1D) spherically symmetric and assumed stationary state. However, the merger of a DD system is a threedimensional (3D) and dynamical event, so multi-dimensional hydrodynamical simulations are necessary to reach a definite conclusion. Benz et al. (1990) used 3D smoothed particle hydrodynamics (SPH) code and simulated mergers of two $\mathrm{CO}$ WDs. Following this work, there were several studies on mergers of DD systems (Rasio \& Shapiro 1995; Segretain \& Chabrier 1997; Guerrero et al. 2004; Yoon et al. 2007; LorénAguilar et al. 2009; Fryer et al. 2010; Pakmor et al. 2010; Dan et al. 2011; Raskin et al. 2012, 2014; Moll et al. 2014). These studies concluded that some DD pairs can explode as $\mathrm{SNe}$ Ia. Such successful models can be divided by the dynamical phase when the SN Ia explosion occurs. 
Pakmor et al. (2010) simulated mergers of massive CO WDs $\left(\sim 0.9 M_{\odot}\right)$ and found that carbon detonation initiates during the dynamical merger phase because of the compressional heating by the disrupted secondary which violently accretes onto the primary. The binary system finally explodes as a subluminous SN Ia. Pakmor et al. (2012a) also simulated mergers of more massive CO WDs $\left(1.1+0.9 M_{\odot}\right)$ and found that the system leads to a normal SN Ia.

If carbon detonation does not initiate in the dynamical merger phase, the remnant undergoes three phases. The first phase is the early remnant phase (e.g., Shen et al. 2012; Kashyap et al. 2015), 100-1000 s after the secondary is completely disrupted. In this phase, the merger remnant does not reach a quasi-stationary state yet and still has small nonaxisymmetric structures. The second phase is the viscous evolution phase (Schwab et al. 2012; Shen et al. 2012; Ji et al. 2013), $10^{4}-10^{8} \mathrm{~s}$ after merging. In this phase, the remnant reaches a quasi-stationary, axisymmetric state and it evolves in a viscous timescale. The third is the thermal evolution phase (Saio \& Nomoto 1985, 2004; Yoon et al. 2007; Shen et al. 2012), $\gtrsim 10^{3}$ years. If off-center carbon burning occurs in these three phases before the rotating core of the remnant reaches $M_{\mathrm{Ch}}$, it likely converts the CO WD to an oxygenneon-magnesium (ONeMg) WD. The ONeMg WD finally collapses to a neutron star when its core mass reaches $M_{\mathrm{Ch}}$. Yoon et al. (2007) simulated the merger of CO WDs with masses of $0.9+0.6 M_{\odot}$ and followed the evolution of the merger remnant. They found that the remnant can avoid offcenter carbon burning and explodes as an SN Ia in the thermal evolution phase, if it satisfies several conditions.

Thus, these works concluded that some DD systems can become SNe Ia. However, the mass range of CO WDs that lead to $\mathrm{SNe}$ Ia is not clarified yet. In this work, we simulate mergers of CO WDs with a mass range of $0.5-1.1 M_{\odot}$ with our SPH code until the end of the early remnant phase, and identify the mass range of CO WDs that lead to SNe Ia. Using the obtained mass range of $\mathrm{CO}$ WDs, we estimate the contribution of CO WD mergers to the entire SNe Ia in our galaxy.

Similar parameter surveys have already been done by Dan et al. (2012, 2014) and Zhu et al. (2013). Zhu et al. (2013) mainly focused on the (early) remnant phase and the resolutions of their simulations are lower than our study. Although Dan et al. $(2012,2014)$ covered the merger phase and remnant phase, their numerical resolution is much lower than ours. We expect that SN Ia explosions occur not only in the early remnant phase but also in the dynamical merger phase. In the present work, we adopt higher resolution than two times those of the previous works (e.g., Zhu et al. 2013). This is because the numerical resolution is one of the most important parameters to identify the initiation of detonation in the dynamical merger phase (Pakmor et al. 2012b). Therefore, we adopt four different resolutions and check the numerical convergence.

To investigate the possibility that carbon burning leads to an SN Ia, we check the density and temperature of SPH particles and identify carbon burning by the condition of $\tau_{\mathrm{CC}}<\tau_{\text {cool }}$, where $\tau_{\mathrm{CC}}$ is the timescale of carbon burning and $\tau_{\text {cool }}$ is the cooling timescale. In the dynamical merger phase, $\tau_{\text {cool }}$ is the dynamical timescale of adiabatic expansion. On the other hand, in the (early) remnant phase, $\tau_{\text {cool }}$ is the timescale of neutrino cooling. These are necessary but not sufficient conditions for a thermonuclear explosion. In this sense, our results would not be conclusive but are sufficiently suggestive. Since the temperature has numerical noise in our SPH simulation, we have to treat the temperature carefully. This noise comes from fluctuations of density and internal energy, which arise due to numerical resolution, finite neighbor particles, and the accuracy of time-integration. We use both the raw and smoothed temperatures to estimate the noise in our SPH simulation (Dan et al. 2012). The raw temperature is the temperature that our SPH code originally generates. The smoothed temperature is an averaged temperature calculated from neighbor particles, which could avoid large numerical noises (defined in Section 3.1). In the present version of our SPH simulation, we do not include nuclear reactions because they are so sensitive to temperature noises and possibly enhance them erroneously.

This paper is organized as follows. Section 2 briefly describes our numerical methods. In section 3 , we show our results and then estimate the rate of $\mathrm{SNe}$ Ia coming from the DD merger systems and their contribution to the entire SNe Ia in our galaxy. In section 4 , we compare our results with previous works and discuss the dependence of our numerical results on the resolution. Finally we conclude the present work in Section 5.

\section{METHODS}

Here, we present a brief summary of our numerical method. The details of the numerical method are already described in Nakasato et al. (2012) and Tanikawa et al. (2015).

\subsection{Numerical Code}

SPH is the Lagrangian mesh-free particle method developed for the study of astrophysical fluid phenomena (Lucy et al. 1977; Gingold \& Monaghan 1982). Nowadays, it is applied to more various subjects, e.g., engineering, meteorology, and Hollywood movies. Recent good reviews about SPH codes are available, e.g., in Monaghan (2005) and Rosswog (2009). Our formulation is the one called "vanilla ice" SPH formulation in Rosswog (2009). Our basic SPH equations consist of the equation of continuity, equation of motion, and energy equation for self-gravitating fluid in Lagrangian formulation.

We use "OcTree On OpenCL"(OTOO) code for our 3D simulations of CO WD mergers, which is developed for various particle simulations of astrophysical fluid phenomena (Nakasato et al. 2012). This code implements the octree method (Barnes \& Hut 1986) to calculate gravity and neighbor particles for SPH. It is optimized for multiple CPUs and GPUs in heterogeneous computational resources.

In this code, we adopt the 3rd-order spline kernel and its derivative is modified in the same way as that proposed by Thomas \& Couchman (1992) to avoid the pairing instability. The smoothing length is determined to keep the average number of neighbors being about 75 in every step (Thacker et al. 2000). The formulation of the artificial viscosity is basically the same as Monaghan (1992), but the viscosity coefficient is time-dependent (Morris \& Monaghan 1997). More detail explanation can be seen in Rosswog et al. (2000) and Monaghan (2005). We also introduce the Balsara switch to shut off the artificial viscosity in no shock regions (Balsara 1995). We adopt a leap-frog scheme for time integration.

We use the Helmholtz equation of state (EOS) (Timmes \& Swesty 2000) and assume a uniform chemical composition of $50 \%$ carbon and $50 \%$ oxygen. This chemical composition is not changed throughout the merger simulation because we do not include nuclear reactions. 


\subsection{Initial Setup}

The initial setup of our simulations is similar to those of Rasio \& Shapiro (1995) and Dan et al. (2011). We separately generate each CO WD from spherically symmetric density profiles of a perfectly degenerate star, and set the temperature at $10^{6} \mathrm{~K}$ everywhere. To reduce numerical noise in the mapping from the 1D spherically symmetric density profile to our 3D SPH density distribution, we relax the SPH particles for 20 physical seconds with velocity damping force but without the evolution of internal energy. For the damping force, we adopt

$$
\frac{d \mathbf{v}_{i}}{d t}=-\frac{\mathbf{v}_{i}}{C_{\mathrm{damp}} d t},
$$

where $\mathbf{v}_{i}$ is the velocity of $i$ th particle, $C_{\text {damp }}$ is the inverse of relaxation timescale and we fix $C_{\text {damp }}=128.0$.

Next, we put two CO WDs to the distance enough to avoid the Roche lobe over flow (RLOF). We assume that they are on a circular orbit and their spins synchronize each other with the orbital motion. To finish setting the initial condition, we gradually decrease the separation and relax them with the above damping force and the evolution of internal energy in a corotating frame. When an SPH ( $i$ th) particle of the secondary approaches closely enough the L1 point, i.e.,

$$
\left\|\mathbf{r}_{\mathrm{sec}, i}-\mathbf{r}_{\mathrm{L} 1}\right\|<0.2 R_{2}
$$

we stop the calculation. Here $\mathbf{r}_{\mathrm{sec}, i}$ is the position of $i$ th particle of the secondary, $\mathbf{r}_{\mathrm{L} 1}$ is that of $\mathrm{L} 1$ point, and $R_{2}$ is the effective radius of the secondary.

Finally, we transfer the SPH particles from the co-rotating frame to a rest frame.

\section{RESULTS}

We summarize the results of our simulations in Table A1. Because we aim to identify the mass range of CO WDs that lead to an SN Ia, our initial models cover the entire mass range of CO WDs, i.e., from $M_{\mathrm{WD}}=0.5$ to $1.1 M_{\odot}$. We prepare binary models of $0.5,0.6,0.7,0.8,0.9,1.0$, and $1.1 M_{\odot}$, and perform the simulations of mergers for all 28 mass combinations. Since we also investigate the dependence of our results on the numerical resolution, we perform the same simulations with different resolutions, which are $10 k, 50 k, 100 k, 500 k$ SPH particles per one solar mass (here $k \equiv 1,024)$. All simulations were performed from the start of RLOF until the formation of almost stationary remnant, several hundred seconds after the secondary is completely disrupted. We divide the dynamical evolution of a merger into the following two phases. (1) The merger phase is the period during which the secondary dynamically accretes onto the primary. (2) The (early) remnant phase is from the complete disruption of the secondary to the stage that the system reaches almost a quasi-stationary state.

Figure 1 shows the density profiles in the equatorial plane for an example of our simulations, whose mass combination is $1.1+0.9 M_{\odot}$ and resolution is $500 k M_{\odot}^{-1}$. The merger phase covers the first $(t=25 \mathrm{~s})$ to fifth $(t=135 \mathrm{~s})$ panels, and the early remnant phase corresponds to the sixth $(t=240 \mathrm{~s})$ panel. Their morphological structures are consistent with the previous works (e.g., Pakmor et al. 2012a).

First we check whether dynamical carbon burning starts or not in the merger phase for all simulations because it is a necessary condition for an SN Ia explosion. Then, we check steady carbon burning in the early remnant phase. If it occurs in the early remnant phase, carbon burning converts a
CO WD into an ONeMg WD and the merger remnant will not become an SN Ia. Although we perform simulations with four different resolutions, we first focus on the highest resolutions $\left(=500 k M_{\odot}^{-1}\right)$.

\subsection{Merger Phase}

Pakmor et al. (2010) first suggested that CO WD mergers lead to an SN Ia in the merger phase. They called such a model the (carbon-ignited) violent merger (VM) scenario. In this scenario, matter of the secondary violently accretes onto the primary and such violent accretion causes dynamical carbon burning. As a result, detonation wave would be formed and propagate into the primary with converting its carbonoxygen into iron group elements. Finally, the system explodes as an SN Ia. Because our simulations cannot directly resolve the initiation of detonation, we try to judge the occurrence of dynamical carbon burning in the merger phase. This condition is a necessary condition for an SN Ia explosion.

For this purpose, we extract the highest temperature particle in the merger phase for all simulations. The condition for dynamical carbon burning is

$$
\tau_{\mathrm{CC}}<\tau_{\text {dyn }},
$$

where $\tau_{\mathrm{CC}}$ is a carbon burning timescale defined by

$$
\tau_{\mathrm{CC}}=\frac{C_{P} T}{\epsilon_{\mathrm{CC}}},
$$

and $\tau_{\text {dyn }}$ is a dynamical timescale (Nomoto 1982) defined by

$$
\tau_{\text {dyn }}=\frac{1}{\sqrt{24 \pi G \rho}},
$$

where $C_{P}$ is the specific heat at constant pressure, $\epsilon_{\mathrm{CC}}$ is the energy generation rate of carbon burning. We calculate the both timescales for each particle in the merger phase and examine whether the particles satisfy Equation (3). In this work, $C_{P}$ is derived from the Helmholtz EOS of Timmes \& Swesty (2000) and $\epsilon_{\mathrm{CC}}$ is the same as that of Dan et al. (2014), originally proposed by Blinnikov \& Khokhlov (1987). Its formulation is

$$
\epsilon_{\mathrm{CC}}=\rho q_{\mathrm{C}} A_{\mathrm{T}} Y_{\mathrm{C}}^{2} \exp \left(-Q / T_{9 \mathrm{a}}^{1 / 3}+f_{\mathrm{CC}}\right),
$$

where $q_{\mathrm{C}}=4.48 \times 10^{18} \mathrm{erg} \mathrm{mol}^{-1}$ (Blinnikov \& Khokhlov 1987), $\quad A_{\mathrm{T}}=8.54 \times 10^{26} T_{9 \mathrm{a}}^{5 / 6} T_{9}^{-3 / 2} \mathrm{~s}^{-1} \mathrm{~mol}^{-1} \mathrm{~cm}^{3}$, $T_{9} \equiv T / 10^{9} \mathrm{~K}, T_{9 \mathrm{a}} \equiv T_{9} /\left(1+0.067 T_{9}\right), Q=84.165$ (Fowler et al. 1975). The carbon abundance is calculated as $Y_{\mathrm{C}}=n_{\mathrm{C}} /\left(\rho N_{\mathrm{a}}\right)=0.033 \mathrm{~mol} \mathrm{~g}^{-1}$, where $n_{\mathrm{C}}$ is the number density of carbon and $N_{\mathrm{a}}$ is the Avogadro number. A screening factor $f_{\mathrm{CC}}$ is ignored here, because we focus on the start of dynamical carbon burning and the factor of self-acceleration for nuclear burning described in Frank-Kamenetskii (1967) is not applied to the initiation of carbon burning.

Figure 2 shows the density and temperature of a particle with the highest temperature in the merger phase for all mass combinations (their resolutions are $500 \mathrm{k} \mathrm{M}_{\odot}^{-1}$ ), similar to Figure 12 of Dan et al. (2014). The shapes and colors of symbols indicate the primary's mass and the total mass, respectively. Solid lines indicate $\tau_{\mathrm{CC}}=\tau_{\mathrm{dyn}}$, and dashed lines do $\tau_{\mathrm{CC}}=0.1 \tau_{\mathrm{dyn}}$. For the mass combinations above the solid line, dynamical carbon burning occurs in the merger phase. So, the merger of CO WDs would lead to an SN Ia explosion.

In our SPH simulation, physical raw temperature of each particle has numerical noise. So we adopt another definition 

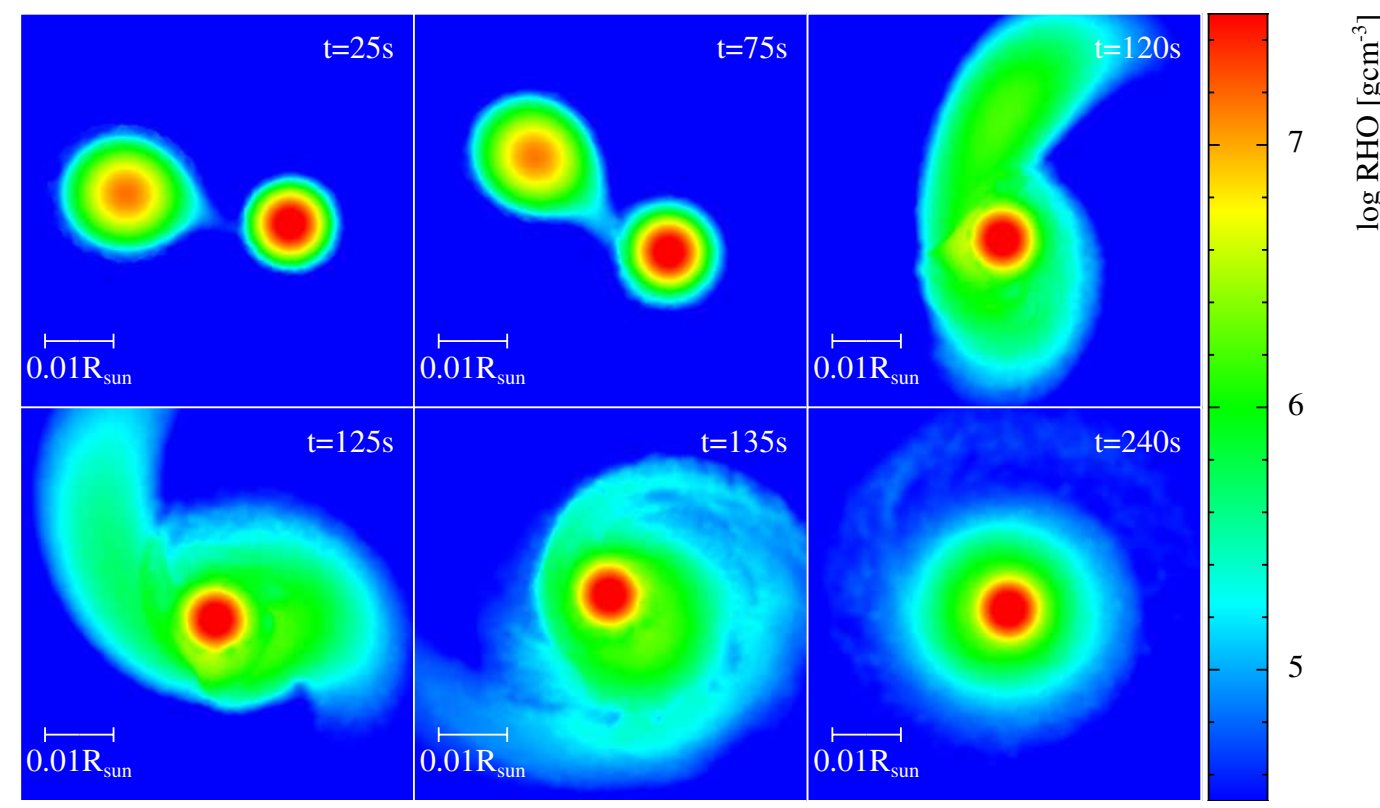

6

5

FIG. 1.- Density profiles in the equatorial plane for the dynamical evolution of our merger simulation. The mass combination is $1.1+0.9 M_{\odot}$, and the resolution is $500 \mathrm{k}_{\odot}^{-1}$. Colors indicate density in a logarithmic scale.

of temperature to reduce the effect of noise, i.e., smoothed temperature. It is defined by

$$
T_{s, i}=\sum_{j} \frac{m_{j}}{\rho_{j}} T_{j} W\left(r_{i j}, h_{i j}\right)
$$

where $m_{j}, \rho_{j}, T_{j}$ are the mass, density, temperature of $j$ th particle, respectively. $r_{i j}=\left\|\mathbf{r}_{i}-\mathbf{r}_{j}\right\|$, and $h_{i j}=\left(h_{i}+h_{j}\right) / 2$ is the average of the smoothing lengths of $i$ th and $j$ th particles.

Figure 2(a) and 2(b) show the results for raw temperature and smoothed temperature thus defined, respectively. It is clear that all symbols in Figure 2(a) move to a lower place in Figure 2(b). As a result, the number of mass combinations above the solid line of $\tau_{\mathrm{CC}}=\tau_{\mathrm{dyn}}$ decreases. Figure 2(b) of smoothed temperature shows that the mass combinations above the solid line would certainly trigger dynamical carbon burning in the merger phase.

\subsection{Remnant Phase}

If dynamical carbon burning does not occur in the merger phase, the merged object goes into a stationary phase, i.e., the (early) remnant phase. Figure 3(a) and 3(b) show the density and temperature, respectively, of the merger remnant whose mass combination and resolution are $1.1+0.9 M_{\odot}$ and $500 k M_{\odot}^{-1}$. The remnant consists of three components, i.e., a cold core, hot envelope, and outer disk. The structure of such a remnant has been studied in the several works as already mentioned in Section 1. Although there are small differences among these previous works, their results are almost consistent with each other. Our results are also consistent with these previous results.

It has long been discussed that such a hot envelope gradually accrete onto a cold core because of angular momentum loss by some mechanisms (e.g. viscosity or magnetic field). If off-center carbon burning occurs quiescently during such an accretion phase, carbon deflagration waves propagate into the core. At last, the whole core is converted into an $\mathrm{ONeMg}$ WD (e.g., Saio \& Nomoto 1985, 1998, 2004). In such a case, it can not explode as an SN Ia, even if its total mass exceeds $M_{\mathrm{Ch}}$. Instead, it collapses to a neutron star (Nomoto \& Kondo 1991). On the other hand, if off-center carbon burning does not occur, the core remains unchanged as a CO WD and surrounding matter continues to accrete onto the core. When the core mass exceeds $M_{\mathrm{Ch}}$, it explodes as an SN Ia. It is critically important to examine whether carbon burning starts quiescently off-center in the remnant phase.

We examine, in the same way as that of dynamical carbon burning in the merger phase, whether or not off-center carbon burning occurs in the remnant phase. Carbon burning quiescently occurs near the boundary between the cold core and hot envelope, if the condition for carbon burning, i.e.,

$$
\tau_{\mathrm{CC}}<\tau_{\nu}
$$

is satisfied in the remnant phase. Here, $\tau_{\nu}$ is a timescale of neutrino cooling and we use the description of Itoh et al. (1996) for calculating it from the density and temperature of SPH particles. We find the highest temperature particle in the remnant phase for all mass combinations, and examine Equation (8). If the highest temperature particle satisfies the condition, we regard that off-center carbon burning starts and converts the $\mathrm{CO}$ core into an $\mathrm{ONeMg}$ core. Then, the system finally collapses to a neutron star if the total mass exceeds $M_{\mathrm{Ch}}$. On the other hand, if there are no particles that satisfy the condition and the total mass of the remnant exceeds $M_{\mathrm{Ch}}$, we consider that the remnant becomes an SN Ia.

Figure 4 is the same plot as Figure 2, but for the remnant phase. Magenta solid lines indicate $\tau_{\mathrm{CC}}=\tau_{\nu}$. Figure 4(a) and 4(b) show results of the raw and smoothed temperatures, respectively. Off-center carbon burning occurs in the remnant phase for the mass combination models above the magenta solid line. They would finally collapse to a neutron star. On the other hand, the models below the line would become an SN Ia if the total mass exceeds the Chandrasekhar mass, i.e., $M_{1}+M_{2}>M_{\mathrm{Ch}}$.

We have to follow the viscous and thermal evolution phases 

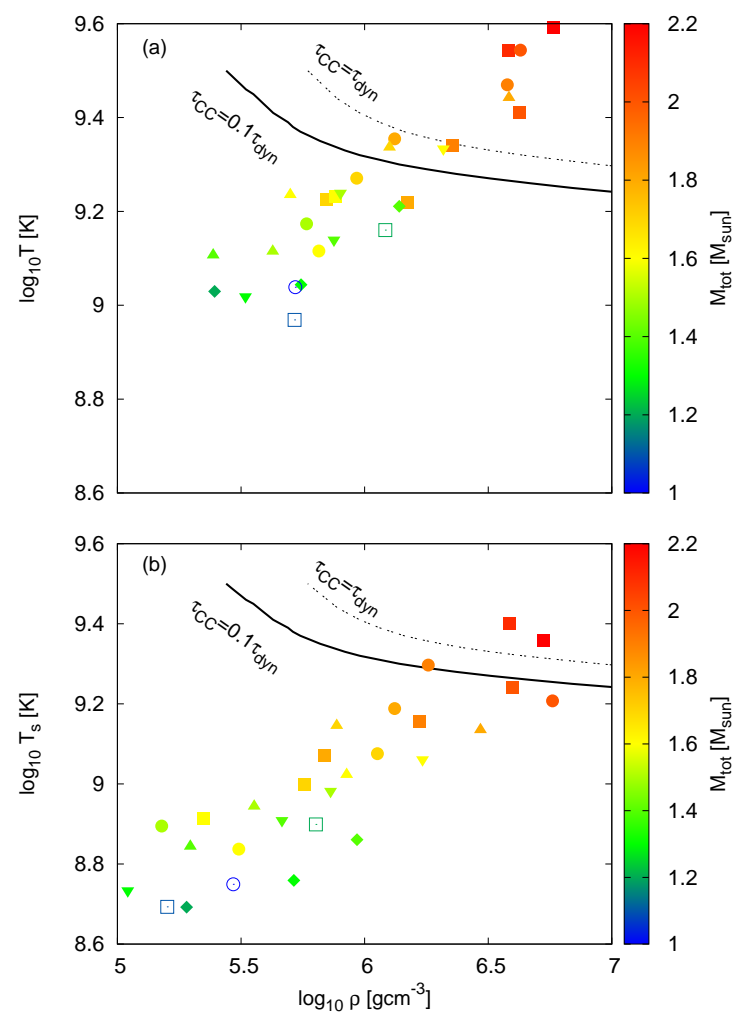

FIG. 2.- Density and temperature of the highest temperature particle in the merger phase for all mass combinations. Numerical resolutions of these models are $500 \mathrm{k} \mathrm{M}_{\odot}^{-1}$. Colors of symbols indicate the total mass of the system as indicated in the rightside of the figures, and shapes of symbols do the mass of the primary. Filled squares are the $1.1 M_{\odot}$ primary, filled circles 1.0 $M_{\odot}$, filled triangles $0.9 M_{\odot}$, filled inverted triangles $0.8 M_{\odot}$, filled diamonds $0.7 M_{\odot}$, open squares $0.6 M_{\odot}$, open circles $0.5 M_{\odot}$. Solid lines indicate $\tau_{\mathrm{CC}}=\tau_{\mathrm{dyn}}$, and dashed lines do $\tau_{\mathrm{CC}}=0.1 \tau_{\mathrm{dyn}}$. (a) Raw temperature of SPH particles. (b) Smoothed temperature, $T_{\mathrm{S}}$, of SPH particles defined by Equation (7).

for obtaining definite conclusion on the off-center carbon burning. However, we stop our SPH calculation at the end of the early remnant phase because our SPH code includes no physical viscosities (e.g., magnetic viscosity). In the viscous and thermal evolution phases, the hot envelope further accretes onto the cold core and compress itself on the cold core. The temperature and density might further increase. As a result, carbon ignites off-center even for the cases of no offcenter burning in the early remnant phase. In fact, Shen et al. (2012) and Schwab et al. (2012) followed the evolution of merger remnants and showed that off-center carbon ignition starts in the viscous and thermal evolution phases for some cases. Yoon et al. (2007) performed SPH simulation of CO WD merger whose mass combination is $0.9+0.6 M_{\odot}$ and further followed the evolution of the merger remnant with a 1D stellar evolution code. They found that off-center carbon burning can be avoided when the highest temperature is lower than the threshold for carbon ignition, angular momentum loss occurs with a timescale longer than that of neutrino cooling, and the mass accretion rate is $\dot{M} \leq 5 \times 10^{-6}$ to $10^{-5} M_{\odot} \mathrm{yr}^{-1}$. Our present condition for off-center carbon burning is posed only for the (early) remnant phase but not applied yet for the viscous and thermal accretion phases. In this sense, our results only for the early remnant phase are not def-
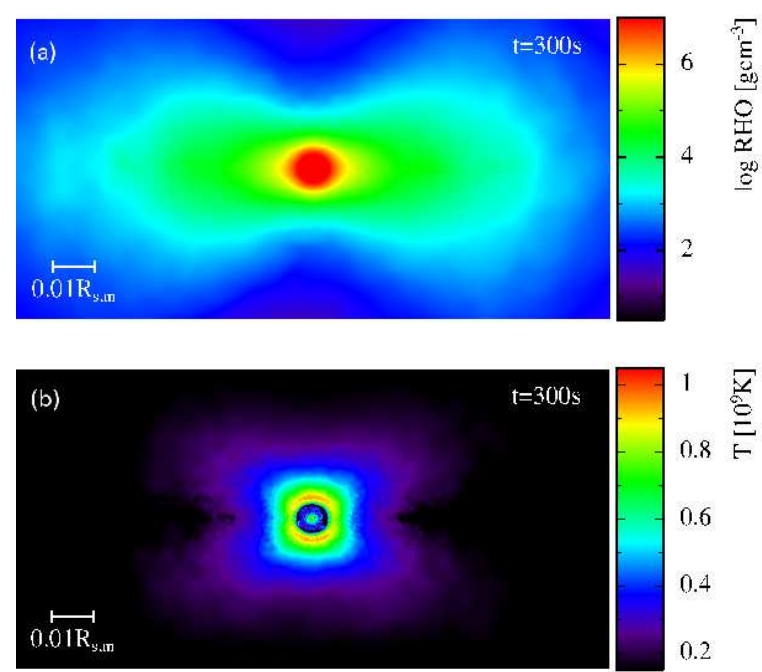

FIG. 3.- Structure of a merger remnant at about $200 \mathrm{~s}\left(\sim 7.6 P_{\text {orb,init }}\right)$ after the secondary is disrupted completely. Here, $P_{\text {orb,init }}$ is the initial orbital period. Its mass combination is $1.1+0.9 M_{\odot}$ and the numerical resolution is $500 \mathrm{k} \mathrm{M}_{\odot}^{-1}$, which is the same model as in Figure 1. (a) Density profile (in a logarithmic scale of $\mathrm{g} \mathrm{cm}^{-3}$ ) in the $x--z$ plane. (b) Temperature structure (in a linear scale of $10^{9} \mathrm{~K}$ ) in the $x--z$ plane. The central part becomes slightly hot. This is caused by numerical noise and artificial viscosity, so it has no physical meaning.

inite answers. We leave such viscous and thermal evolutions of the merger remnants in our future works.

\subsection{White Dwarf Mass Combinations For SNe Ia}

Now, we have obtained the mass range of CO WDs which possibly lead to an SN Ia. Using this mass range of $\mathrm{CO}$ WDs, we estimate their contribution to the entire SNe Ia in our galaxy. We consider four paths that CO WD mergers would follow. The first one is the VM path, the condition of which is that dynamical carbon burning occurs in the merger phase. The systems satisfying this condition would explode as an SN Ia immediately after merging (Pakmor et al. 2010, 2011, 2012a). When dynamical carbon burning does not occur in the merger phase, the system enters the remnant phase and the disrupted secondary surrounds the primary. If its total mass exceeds $M_{\mathrm{Ch}}$ and off-center carbon burning does not occur during the accretion phase, carbon burning occurs at the center of the CO WD and it would finally explode as an SN Ia. We regard this evolutionary path as the accretion induced explosion (AIE) path. On the other hand, if off-center carbon burning occurs, the core of the remnant will be converted into an $\mathrm{ONeMg} \mathrm{WD}$ and then collapse to a neutron star when the core mass exceeds $M_{\mathrm{Ch}}$. This is the accretion induced collapse (AIC) path (Saio \& Nomoto 1985, 1998, 2004; Nomoto $\&$ Kondo 1991). When dynamical carbon burning does not occur in the merger phase and the total mass of the system does not exceed $M_{\mathrm{Ch}}$, the system would form a massive $\mathrm{CO}$ WD. We call this evolutionary path as the massive white dwarf (MWD) path.

Figure 5 shows all mass combinations of our simulations with $500 k M_{\odot}^{-1}$ particles and identifies which path they take. Colors of symbols indicate the four paths, i.e., the VM (red), AIE (green), AIC (blue), and MWD (magenta) paths. Among these four paths, the VM and AIE paths are possible paths to $\mathrm{SNe} \mathrm{Ia}$. 

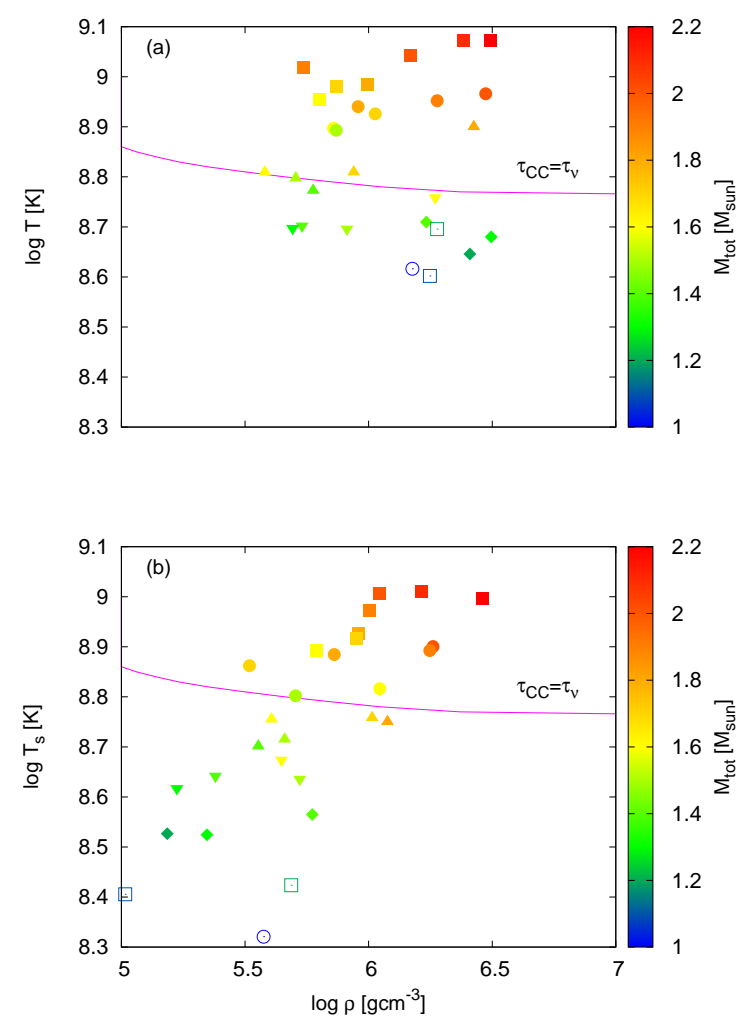

FIG. 4.- Same as Figure 2, but for the remnant phase. Magenta solid lines indicate $\tau_{\mathrm{CC}}=\tau_{\nu}$.

\subsection{SN Ia Rate}

We estimate the rate of $\mathrm{SNe}$ Ia which originate from $\mathrm{CO}$ WD mergers and their contribution to the entire SNe Ia in our galaxy. Here, we assume that all mergers of CO WDs satisfying the VM or AIE condition can explode as an SN Ia. Strictly speaking, for the VM path, dynamical carbon burning is a necessary, but not a sufficient condition for carbon detonation. So it is not trivial if those mergers explode as an SN Ia. On the other hand, we have to follow the evolution of merger remnants for a much longer time (e.g., Yoon et al. 2007) in order to identify their final fates, the AIE (exploding as an SN Ia) or AIC (collapsing to a neutron star) path. In this sense, our estimate is just an upper limit.

The SN Ia rate depends on the Hubble type and stellar mass of a galaxy. According to Li et al. (2011), in SBc type galaxies with similar stellar mass to our galaxy, the SN Ia rate is about $1.1 \times 10^{-13} \mathrm{yr}^{-1} M_{\odot}^{-1}$. Badenes et al. (2012) estimated the merger rate of binary WDs in our galaxy as $1.4 \times 10^{-13} \mathrm{yr}^{-1} M_{\odot}^{-1}$. The mass distribution of binary WDs in our galaxy is still uncertain because there are small samples even in our neighborhood. We assume that both the primary and the secondary follow the mass distribution of single DA WDs in our galaxy derived from SDSS-DR7 (see, e.g., Figure 10 of Kleinman et al. 2013). Then we can calculate the rate of CO WD mergers that satisfy the condition of each scenario.

The merger rate is about $1.4 \times 10^{-15} \mathrm{yr}^{-1} M_{\odot}^{-1}$ $\left(0.14 \times 10^{-15} \mathrm{yr}^{-1} M_{\odot}^{-1}\right)$ for the $\mathrm{VM}, 5.4 \times 10^{-15} \mathrm{yr}^{-1} M_{\odot}^{-1}$ $\left(8.9 \times 10^{-15} \mathrm{yr}^{-1} M_{\odot}^{-1}\right) \quad$ for the AIE path, and $6.8 \times 10^{-15} \mathrm{yr}^{-1} M_{\odot}^{-1}\left(9.0 \times 10^{-15} \mathrm{yr}^{-1} M_{\odot}^{-1}\right)$ for the both
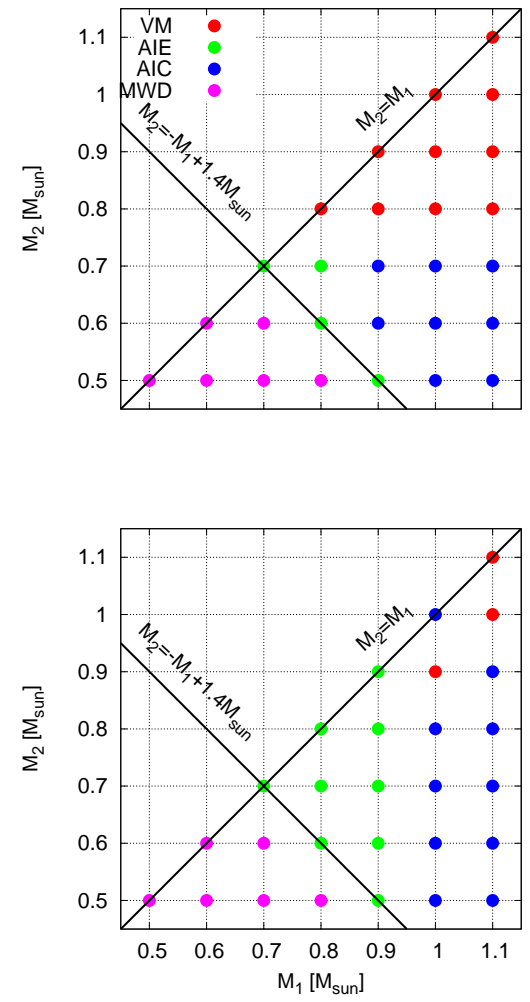

FIG. 5.- The outcome of our merger simulations for the (a) raw and (b) smoothed temperatures. Red symbols mean the VM path, green symbols the AIE path, blue the AIC, and magenta the MWD path. Two black lines indicate that the primary and secondary have the same mass and that the total mass equals $M_{1}+M_{2}=M_{\mathrm{Ch}} \sim 1.4 M_{\odot}$. Mass combinations of red and green symbols result in an SN Ia.

paths, if we adopt the case of raw temperature (smoothed temperature). This is only $\lesssim 9 \%$ of the entire galactic SNe Ia. Therefore, at least in our galaxy, DD merger systems might not dominate the progenitors of SNe Ia.

\section{DISCUSSION}

\subsection{Comparison With Previous Studies}

We compare the present results with previous studies. In particular, we mainly focus on two mass combinations. One is $1.1+0.9 M_{\odot}$ and the other is $0.9+0.6 M_{\odot}$, because these two combinations were well studied in the previous works.

$$
\text { 4.1.1. } 1.1+0.9 M_{\odot}
$$

First we compare our result of $1.1+0.9 M_{\odot}$ and $100 k M_{\odot}^{-1}$ (i.e. the total number of SPH particles is about $2 \times 10^{5}$ ) with that of Pakmor et al. (2012b). Figure 6(a) shows the time evolutions of the orbital separation and Figure 6(b) shows the number of particles having temperature higher than $2 \times 10^{9} \mathrm{~K}$. Our Figure 6 should be compared with Figure 4 and Table 1 of Pakmor et al. (2012b).

It should be noted that the WDs in our model merge more quickly (about $100 \mathrm{~s}$ ) than those in Pakmor et al. (2012b) (about $600 \mathrm{~s}$ ) although we start our simulation with the initial condition similar to theirs. This is because our initial separation $\left(\sim 1.67 \times 10^{9} \mathrm{~cm}\right)$ is less than Pakmor et al. (2012b) $\left(\sim 1.93 \times 10^{9} \mathrm{~cm}\right)$. Indeed, Figure 6(a) and 6(b) resemble the case of their smaller initial separation. We suppose that the 

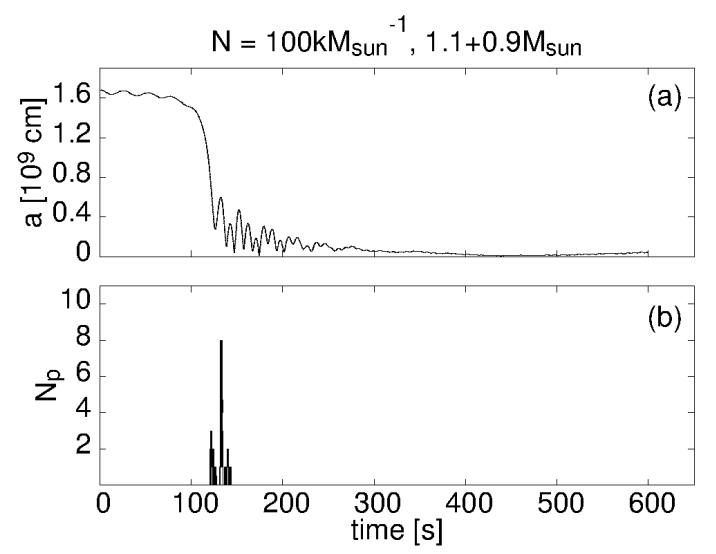

FIG. 6.- Time evolution of (a) the orbital separation and (b) the number of high temperature $\left(\geq 2.0 \times 10^{9} \mathrm{~K}\right)$ particles. The mass combination of WDs is $1.1+0.9 M_{\odot}$ and the numerical resolution is $100 k M_{\odot}^{-1}$.

main reason of the difference is the relaxation method of a single WD. As mentioned in Section 2.2, we relax a single WD with a velocity-dependent damping force but without the evolution of internal energy. On the other hand, Pakmor et al. (2012b) changed the damping timescale (see their Equation 14). After the relaxation method of Pakmor et al. (2012b) is applied to our models, the radii of the relaxed WDs are a few percent larger than our original ones. Therefore, their separations when the RLOF starts are larger than ours and it results in a longer merging time.

If the initial separation is smaller when the RLOF starts, the mass transfer tends to occur more violently and the secondary is completely disrupted in a few orbital periods (Dan et al. 2011). As a result, accreted matter is more strongly shockheated and dynamical carbon burning easily occurs. Although Pakmor et al. (2012b) concluded that the initial condition is not so important for dynamical carbon burning, we should note that our highest temperatures in the merger phase could be overestimated.

$$
\text { 4.1.2. } 0.9+0.6 M_{\odot}
$$

We also compare our result of $0.9+0.6 M_{\odot}$ and $100 k M_{\odot}^{-1}$ with the previous studies of Yoon et al. (2007), Dan et al. (2011), and Zhu et al. (2013). Since these studies have similar resolution (a few $\times 10^{5} \mathrm{SPH}$ particles in all), it is suitable for comparison. Figure 7 shows the time evolution of highest temperature in each density zone. Since no dynamical carbon burning occurs (see Figure 5), we examine whether or not quiescent carbon burning occurs in the remnant phase, i.e., at $t \sim 300 \mathrm{~s}$ in Figure 7. The temperature is about $6 \times 10^{8} \mathrm{~K}$ and this is consistent with the above three previous studies.

We compare our Figure 7 with Figure 4 in Yoon et al. (2007). For the highest temperature in the merger phase (at $t \sim 100 \mathrm{~s}$ in Figure 7 ), their results of $1.7 \times 10^{9} \mathrm{~K}$ is higher than ours $\left(1.3 \times 10^{9} \mathrm{~K}\right)$, although dynamical carbon burning does not occur in the merger phase for both ours and theirs. We suppose that the difference in the highest temperature comes from the difference in the initial condition. Dan et al. (2011)

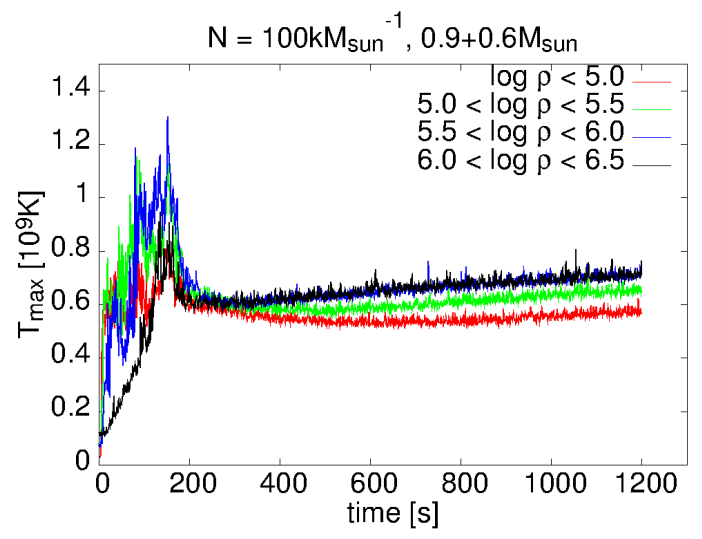

FIG. 7.- Evolution of highest temperature in each density range, $\log \rho\left(\mathrm{g} \mathrm{cm}^{-3}\right)<5.0$ (red), $5.0<\log \rho<5.5$ (green), $5.5<\log \rho<6.0$ (blue), $6.0<\log \rho<6.5$ (black), for the mass combination of $0.9+0.6 M_{\odot}$ and the resolution of $100 \mathrm{k} M_{\odot}^{-1}$.

reported that the morphology of merger remnant could be affected by the initial condition. They found that the remnant whose initial separation is larger has longer trailing arm than the one whose initial separation is smaller (see Figure 10 of Dan et al. 2011). On the other hand, we find that the highest temperature in the remnant phase is barely affected by the initial condition (see Figure 7). Similar discussion appears in Tanikawa et al. (2015).

Other factors might affect the results of our simulations. For example, Zhu et al. (2013) and Dan et al. (2014) performed merger simulations of non-spinning WDs and found that the structures of such merger remnants are different from those in mergers of synchronously spinning WDs, especially for the case of nearly equal masses. In non-spinning cases, the high temperature region is formed near the center of the merger remnant, and carbon burning might occur in that region. Since it is still uncertain whether binary WDs maintain synchronization until their merger, our results for the AIE path might be changed. The topic of synchronization is out of the scope in this paper, so we leave this in our future works.

\subsection{Numerical Resolution}

Pakmor et al. (2012b) concluded that the numerical resolution of simulation is one of the most important factors for carbon burning in the merger phase because very high resolution is required to identify very small hot spots. Therefore, we examine the dependence of our results on the numerical resolution. We perform the same simulations with four different resolutions, i.e., $10 k, 50 k, 100 k$, and $500 k$ per one solar mass. The highest temperature is critical both for dynamical carbon burning and quiescent carbon burning, so we focus on the dependence of the highest temperature on the resolution.

Figure 8(a) and 8(b) show the dependence of the highest temperature in the merger phase on the numerical resolution for the (a) raw and (b) smoothed temperatures, respectively. The highest temperature increases with the number of SPH particles. In other words, our simulation does not converge yet and the final fates of some models could be changed from 

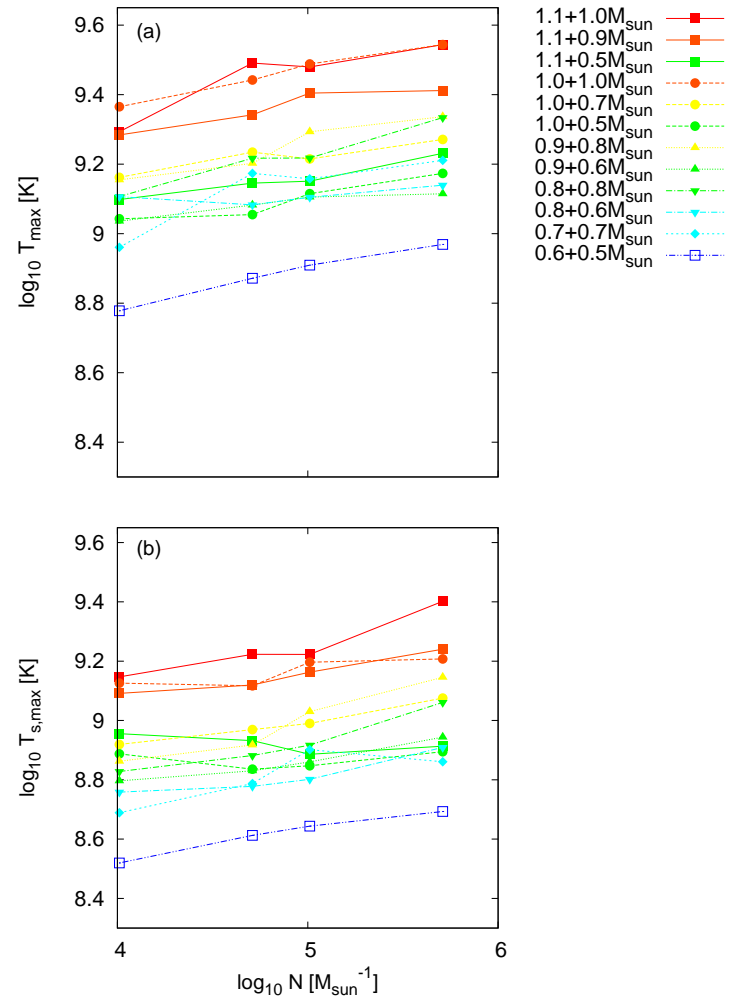

FIG. 8.- Dependence of highest temperature on the numerical resolution in the merger phase for the (a) raw and (b) smoothed temperatures. The horizontal axis is the number of SPH particles per a solar mass. The vertical axis is the highest temperature. Shapes and colors of symbols have the same meaning as those in Figure 2. The highest temperature tends to increase with the numerical resolution.

the AIC path to the VM path. Such a tendency was also reported in Pakmor et al. (2012b). In this sense, we must further increase the numerical resolution to definitely identify the fate of merger products, at least, more than $500 k M_{\odot}^{-1}$. Figure 9(a) and 9 (b) show the dependence of the minimum $\tau_{\mathrm{CC}} / \tau_{\text {dyn }}$ ratio in the merger phase on the numerical resolution for the (a) raw and (b) smoothed temperatures, respectively. The magenta dashed line indicates $\tau_{\mathrm{CC}} / \tau_{\text {dyn }}=1.0$. It tends to decrease as the numerical resolution increases. This trend is consistent with that of the highest temperature. For smoothed temperature, only very massive pairs (both masses $\gtrsim 1.0 M_{\odot}$ ) can ignite carbon dynamically.

On the other hand, Figure 10(a) and 10(b) show the highest temperature in the remnant phase for the (a) raw and (b) smoothed temperatures, respectively. Comparing with the results in the merger phase (Figure 8), the highest temperature depends barely on the resolution. Especially, for the smoothed temperature in Figure 10(b), it converges for almost all the mass combinations. This tendency of weak dependence on the resolution was already reported in the previous studies (Raskin et al. 2012; Dan et al. 2014).

\section{CONCLUSIONS}

We have performed SPH simulations of CO WD mergers for the mass combinations of $0.5-1.1 M_{\odot}$ from the start of the RLOF to the formation of a quasi-stationary merger remnant, and examined whether carbon burning occurs either in the merger phase or remnant phase. Using the results
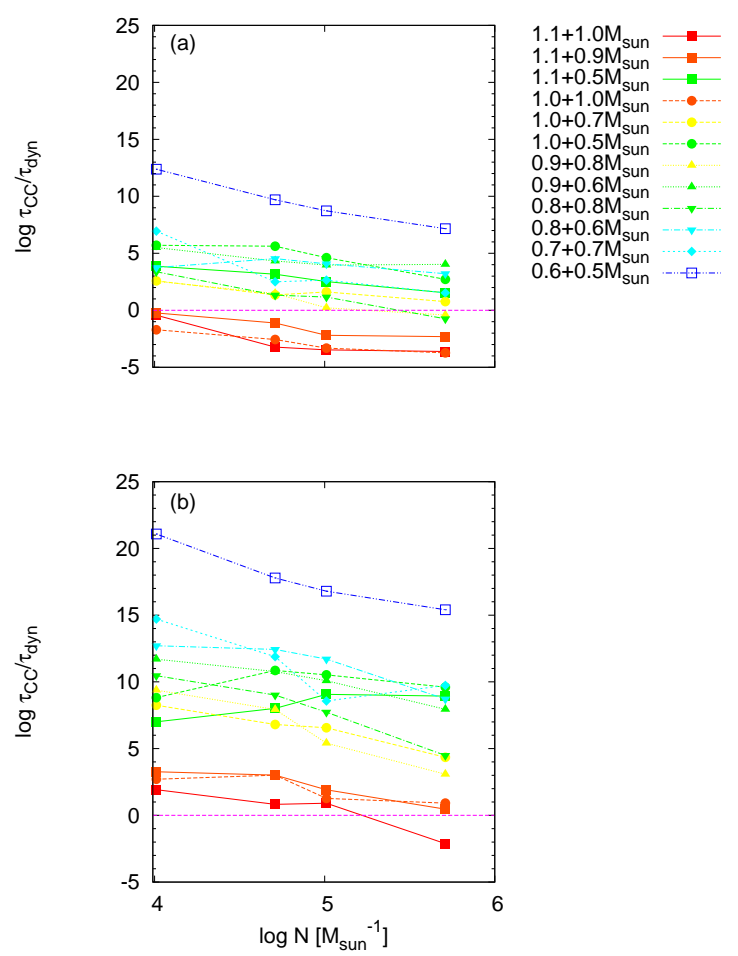

FIG. 9.- Dependence of minimum $\tau_{\mathrm{CC}} / \tau_{\text {dyn }}$ ratio on the numerical resolution in the merger phase for the (a) raw and (b) smoothed temperatures. The horizontal magenta dashed lines indicate $\tau_{\mathrm{CC}} / \tau_{\mathrm{dyn}}=1.0$.

of SPH simulations, we have investigated the mass range of CO WDs that possibly lead to an SN Ia in the merger phase or remnant phase. We have obtained the mass range as follows. When the primary and secondary are as massive as $0.9 M_{\odot} \leq M_{1,2} \leq 1.1 M_{\odot}$, the binary results in an SN Ia in the merger phase. On the other hand, when the primary is $0.7 M_{\odot} \leq M_{1} \leq 0.9 M_{\odot}$ and the total mass of the binary exceeds $1.4 M_{\odot}$, they lead to an SN Ia in the remnant phase.

From the obtained mass range, we have estimated the rate of $\mathrm{SNe}$ Ia coming from $\mathrm{CO}$ WD mergers in our galaxy. It is $6.8 \times 10^{-15} \mathrm{yr}^{-1} M_{\odot}^{-1}$ if we use the results of our raw temperature calculations, while it is $9.0 \times 10^{-15} \mathrm{yr}^{-1} M_{\odot}^{-1}$ if we use that of the smoothed temperature. These are only less than $9 \%$ of the entire SN Ia rate. Therefore, it is unlikely that the mergers of CO WDs are the main progenitors of SNe Ia.

Of course, the above estimate is not conclusive because of several uncertainties in our calculation. We have checked the dependence of the highest temperature on the numerical resolution both in the merger and remnant phases in order to examine the numerical convergence of our simulations. We have found that the highest temperature in the merger phase depends on the numerical resolution. It tends to increase with the resolution as already reported in Pakmor et al. (2012b). On the other hand, in the remnant phase, the highest temperature depends barely on the numerical resolution. Therefore, it is necessary to increase the number of SPH particles, at least, up to $\geq 500 k M_{\odot}^{-1}$, for definite conclusion. Additionally, our calculations for SNe Ia in the remnant phase is not sufficient. In order to obtain the decisive conclusion, we have to follow 

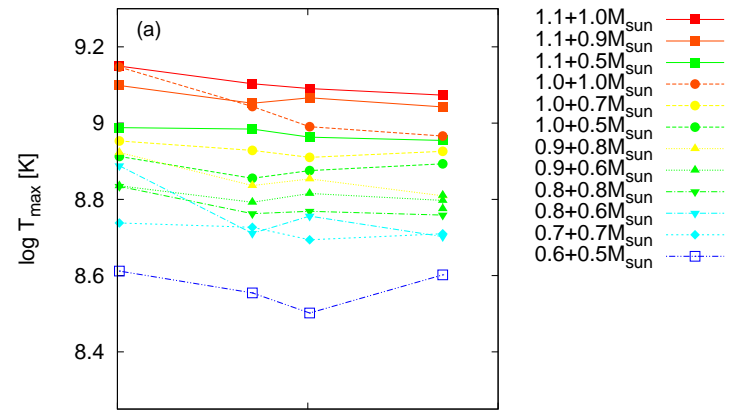

$1.1+0.9 \mathrm{M}_{\text {sun }} \longrightarrow$

$1.1+0.5 \mathrm{M}_{\text {sun }}$
$1.0+1.0 \mathrm{M}_{\text {sun }}$
$1.0+0.7$

$1.0+0.7 \mathrm{M}_{\text {sun }}$

$1.0+0.5 \mathrm{M}$ sun

$0.9+0.8 \mathrm{M}$ su

$0.9+0.6 \mathrm{M}$ sun

$0.8+0.8 \mathrm{M}$ sun

$0.8+0.6 \mathrm{M}_{\text {sun }}$

$0.6+0.5 \mathrm{M}_{\text {sun }}$

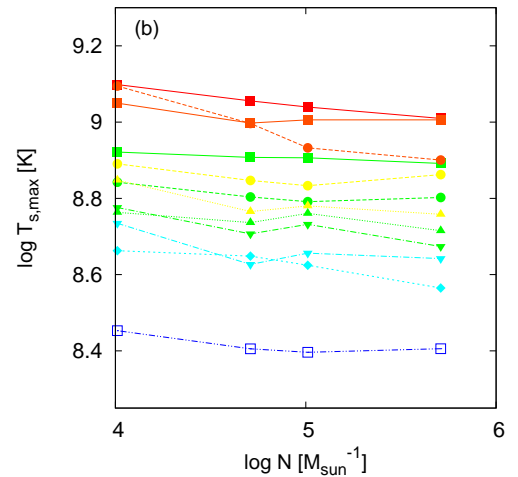

the further evolution of the merger remnant, like Yoon et al. (2007). This is one of our future works.

We thank the anonymous referee for many detailed comments that help to improve the paper. These simulations were performed by using computational resources of Kavli Institute for the Physics and Mathematics of the Universe (IPMU), and HA-PACS at the Center for Computational Sciences in University of Tsukuba under Interdisciplinary Computational Science Program. This research has been supported in part by Grants-in-Aid for Scientific Research (23224004, 23540262, 23740141, 24540227, 26400222, and 26800100) from the Japan Society for the Promotion of Science and by the World Premier International Research Center Initiative, MEXT, Japan. This work is partly supported by MEXT program for the Development and Improvement for the Next Generation Ultra High-Speed Computer System under its Subsidies for Operating the Specific Advanced Large Research Facilities.

FIG. 10.- Same as Figure 8, but for the highest temperature in the remnant phase. The highest temperature seems to converge.

\section{APPENDIX}

\section{SUMMARY OF CO WD MERGER SIMULATIONS}

We summarize our numerical results in Table 1 and 2. The numeric values presented in the table are the primary mass, $M_{1}$, secondary mass, $M_{2}$, initial orbital separation, $a_{\text {init }}$, raw and smoothed maximum temperatures, $T_{\max }$ and $T_{\mathrm{s}, \max }$, densities at the highest raw temperature and at the highest smoothed temperature, $\rho\left(T_{\max }\right)$ and $\rho\left(T_{\mathrm{s}, \max }\right)$, in the merger phase, $T_{\max , \text { rem }}$ and $T_{\mathrm{s}, \mathrm{max}, \text { rem }}, \rho\left(T_{\mathrm{max}, \text { rem }}\right)$ and $\rho\left(T_{\mathrm{s}, \text { max,rem }}\right)$ in the remnant phase.

\section{REFERENCES}

Badenes, C., \& Maoz, D. 2012, ApJ, 749, L11

Balsara, D. S. 1995, JCoPh, 121, 357

Barnes, J., \& Hut, P. 1986, Natur, 324, 446

Benz, W., Cameron, A. G. W., Press, W. H., \& Bowers, R. L. 1990, ApJ, 348,647

Blinnikov, S. I., \& KhoKhlov, A. M. 1987, SvAL, 13, 364

Blondin, S., Prieto, J. L., Patat, F., et al. 2009, ApJ, 693, 207

Dan, M., Rosswog, S., Brüggen, M., \& Podsiadlowski, P. 2014, MNRAS, 438,14

Dan, M., Rosswog, S., Guillochon, J., Ramirez-Ruiz, E. 2011, ApJ, 737, 89

Dan, M., Rosswog, S., Guillochon, J., \& Ramirez-Ruiz, E. 2012, MNRAS, 422, 2417

Di Stefano, R., Voss, R., \& Claeys, J. S. W. 2011, ApJ, 738, L1

Eggleton, P. P. 1983, ApJ, 268, 368

Fowler, W. A., Caughlan, G. R., \& Zimmerman, B. A. 1975, ARA\&A, 13, 69

Fryer, C. L., Ruiter, A. J., Belczynski, K., et al. 2010, ApJ, 725, 296

Frank-Kamenetskii, D. 1967, Diffusion and Heat Transfer in Chemical Kinetic (Moscow: Nauka)

Gingold, R. A., \& Monaghan, J. J. 1982, JCoPh, 46, 429

Guerrero, J., Garcia-Berro, E., \& Isern, J. 2004, A\&A, 413, 257

Hachisu, I., Kato, M., \& Nomoto, K. 1996, ApJ, 470, L97

Hachisu, I., Kato, M., \& Nomoto, K. 1999a, ApJ, 522, 487

Hachisu, I., Kato, M., \& Nomoto, K. 2012, ApJ, 756, L4

Hachisu, I., Kato, M., Nomoto, K., \& Umeda, H. 1999b, ApJ, 519, 314
Iben, I. Jr., \& Tutukov, A. V. 1984, ApJS, 54, 335

Itoh, N., Hayashi, H., Nishikawa, A., \& Kohyama, Y. 1996, ApJS, 102, 411 Ji, S., Fisher, R. T., García-Berro, E., et al. 2013, ApJ, 773, 136 Justhum, S. 2011, ApJ, 730, L34

Kashyap, R., Fisher, R., García-Berro, E., et al. 2015, ApJ, 800, L7

Kleinman, S. J., Kepler, S. O., Koester, D., et al. 2013, ApJS, 204, 5

Kobayashi, C., Tsujimoto, T., Nomoto, K., Hachisu, I., \& Kato, M. 1998, ApJ, 503, L155

Li, W., Chornock, R., Filippenko, A. V., et al. 2011, MNRAS, 412, 1473

Lorén-Aguilar, P., Isern, J., \& Garcia-Berro, E. 2009, A\&A, 500, 1193

Lucy, L. B. 1977, AJ, 82, 1013

Maoz, D., Mannucci, F., \& Nelemans, G. 2014, ARA\&A, 52, 107

Moll, R., Raskin, C., Kasen, D., \& Woosley, S. E. 2014, ApJ, 785, 105

Monaghan, J. J. 1992, ARA\&A, 30, 543

Monaghan, J. J. 2005, RPPh, 68, 1703

Morris, J. P., \& Monaghan, J. J. 1997, JCoPh, 136, 41

Nakasato, N., Ogiya, G., Miki, Y., Mori, M., \& Nomoto, K. 2012, arXiv:1206.1199

Nomoto, K. 1982, ApJ, 257, 780

Nomoto, K., \& Kondo, Y. 1991, ApJ, 367, L19

Pakmor, R., Edelmann, P., Röpke, F. K., \& Hillebrandt, W. 2012b, MNRAS, 424, 2222

Pakmor, R., Hachinger, S., Röpke, F. K., \& Hillebrandt, W. 2011, A\&A, 528, A117

Pakmor, R., Kromer, M., Röpke, F. K., et al. 2010, Natur, 463, 61 
Pakmor, R., Kromer, M., Taubenberger, S., et al. 2012a, ApJ, 747, L10 Patat, F., Chandra, P., Chevalier, R., et al. 2007, Sci, 317, 924

Perlmutter, S., Aldering, G., Goldhaber, G., et al. 1999, ApJ, 517, 565

Price, D. J. 2007, PASA, 24, 159

Rasio, F. A., \& Shapiro, S. L. 1995, ApJ, 438, 887

Raskin, C., \& Kasen, D. 2013, ApJ, 772, 1

Raskin, C., Kasen, D., Moll, R., Schwab, J., \& Woosley, S. E. 2014, ApJ, 788, 75

Raskin, C., Scannapieco, E., Fryer, C., Rockefeller, G., \& Timmes, F. X. 2012, ApJ, 746, 62

Riess, A. G., Fillipenko, A. V., Challis, P., et al. 1998, AJ, 116, 1009

Rosswog, S. 2009, NewAR, 53, 78

Rosswog, S., Davies, M. B., Thielemann, F. K., \& Piran, T. 2000, A\&A, 360,171

Saio, H., \& Nomoto, K. 1985, A\&A, 150, L21

Saio, H., \& Nomoto, K. 1998, ApJ, 500, 388

Saio, H., \& Nomoto, K. 2004, ApJ, 615, 444

Schaefer, B., \& Pagnotta, A. 2012, Natur, 481, 164

Schwab, J., Shen, K. J., Quataert, E., Dan, M., \& Rosswog, S. 2012, MNRAS, 427, 190
Segretain, L., Chabrier, G., \& Mochkovitch, R. 1997, ApJ, 481, 355

Shen, K. J., Bildsten, L., Kasen, D., \& Quataert, E. 2012, ApJ, 748, 35

Shen, K. J., Guillochon, J., \& Foley, R. 2013, ApJ, 770, L35

Simon, J. D., Gal-Yam, A., Gnat, O., et al. 2009, ApJ, 702, 1157

Soker, N., Kashi, A., García-Bero, E., Torres, S., \& Camacho, J. 2013, MNRAS, 431, 1541

Sternberg, A., Gal-Yam, A., Simon, J. D., et al. 2011, Sci, 333, 856

Tanikawa, A., Nakasato, N., Sato, Y., et al. 2015, arXiv:1504.06035

Thacker, R. J., Tittley, E. R., Pearce, F. R., Couchman, H. M. P., \& Thomas,

P. A. 2000, MNRAS, 319, 619

Thomas, P. A., \& Couchman, H. M. P. 1992, MNRAS, 257, 11

Timmes, F. X., \& Swesty, F. D. 2000, ApJS, 126, 501

Webbink, R. F. 1984, ApJ, 277, 355

Whelan, J., \& Iben, I., Jr. 1973, ApJ, 186, 1007

Yoon, S. C., Podsiadlowski, P., \& Rosswog, S. 2007, MNRAS, 380, 933

Zhu, C., Chang, P., van Kerkwijk, M. H., \& Wadsley, J. 2013, ApJ, 767, 164 
TABLE 1

SUMMARY OF ALL CALCULATED MODELS

\begin{tabular}{|c|c|c|c|c|c|c|c|c|c|c|}
\hline $\begin{array}{c}M_{1} \\
\left(M_{\odot}\right)\end{array}$ & $\begin{array}{c}M_{2} \\
\left(M_{\odot}\right)\end{array}$ & $\begin{array}{c}a_{\text {init }} \\
\left(10^{9} \mathrm{~cm}\right)\end{array}$ & $\begin{array}{c}T_{\max } \\
\left(10^{9} \mathrm{~K}\right)\end{array}$ & $\begin{array}{c}\rho\left(T_{\max }\right) \\
\left(10^{6} \mathrm{~g} \mathrm{~cm}^{-3}\right)\end{array}$ & $\begin{array}{c}T_{\mathrm{s}, \max } \\
\left(10^{9} \mathrm{~K}\right)\end{array}$ & $\begin{array}{c}\rho\left(T_{\mathrm{s}, \max }\right) \\
\left(10^{6} \mathrm{~g} \mathrm{~cm}^{-3}\right)\end{array}$ & $\begin{array}{l}T_{\max , \text { rem }} \\
\left(10^{9} \mathrm{~K}\right)\end{array}$ & $\begin{array}{c}\rho\left(T_{\max , \text { rem }}\right) \\
\left(10^{6} \mathrm{~g} \mathrm{~cm}^{-3}\right)\end{array}$ & $\begin{array}{c}T_{\mathrm{s}, \max , \mathrm{rem}} \\
\left(10^{9} \mathrm{~K}\right)\end{array}$ & $\begin{array}{l}\rho\left(T_{\mathrm{s}, \max , \mathrm{rem}}\right) \\
\left(10^{6} \mathrm{~g} \mathrm{~cm}^{-3}\right)\end{array}$ \\
\hline \multicolumn{11}{|c|}{ Resolution $=10 \mathrm{kM}_{\odot}{ }^{-1}$} \\
\hline 1.1 & 1.1 & 1.22 & 2.66 & 6.72 & 1.94 & 7.57 & 1.81 & 2.72 & 1.69 & 3.12 \\
\hline 1.1 & 1.0 & 1.42 & 1.96 & 3.65 & 1.40 & 9.52 & 1.41 & 1.94 & 1.25 & 2.52 \\
\hline 1.1 & 0.9 & 1.64 & 1.92 & 3.37 & 1.23 & 6.59 & 1.25 & 3.02 & 1.12 & 2.98 \\
\hline 1.1 & 0.8 & 1.86 & 1.86 & 1.83 & 1.10 & 4.00 & 1.18 & 2.94 & 1.02 & 2.37 \\
\hline 1.1 & 0.7 & 2.11 & 1.45 & 0.87 & 1.02 & 3.90 & 1.25 & 1.82 & 0.94 & 2.74 \\
\hline 1.1 & 0.6 & 2.40 & 1.40 & 1.21 & 0.92 & 3.20 & 1.01 & 2.64 & 0.88 & 2.48 \\
\hline 1.1 & 0.5 & 2.75 & 1.25 & 1.10 & 0.90 & 1.68 & 0.97 & 1.03 & 0.83 & 2.29 \\
\hline 1.0 & 1.0 & 1.40 & 2.31 & 4.67 & 1.33 & 4.11 & 1.40 & 3.12 & 1.24 & 3.03 \\
\hline 1.0 & 0.9 & 1.61 & 1.54 & 3.24 & 0.94 & 3.26 & 1.05 & 2.35 & 0.94 & 2.16 \\
\hline 1.0 & 0.8 & 1.81 & 1.73 & 2.16 & 0.88 & 2.93 & 0.93 & 2.50 & 0.83 & 2.01 \\
\hline 1.0 & 0.7 & 2.05 & 1.45 & 1.08 & 0.83 & 0.75 & 0.90 & 2.12 & 0.78 & 2.51 \\
\hline 1.0 & 0.6 & 2.35 & 1.35 & 1.10 & 0.79 & 1.01 & 0.82 & 2.78 & 0.74 & 2.52 \\
\hline 1.0 & 0.5 & 2.68 & 1.10 & 0.32 & 0.77 & 1.50 & 0.82 & 3.08 & 0.70 & 1.88 \\
\hline 0.9 & 0.9 & 1.62 & 1.75 & 2.12 & 0.89 & 1.20 & 0.99 & 2.83 & 0.89 & 1.20 \\
\hline 0.9 & 0.8 & 1.79 & 1.43 & 1.52 & 0.73 & 2.02 & 0.84 & 2.58 & 0.70 & 1.55 \\
\hline 0.9 & 0.7 & 2.06 & 1.23 & 1.91 & 0.73 & 1.30 & 0.72 & 2.07 & 0.61 & 1.98 \\
\hline 0.9 & 0.6 & 2.34 & 1.09 & 0.62 & 0.63 & 0.65 & 0.68 & 1.17 & 0.58 & 1.37 \\
\hline 0.9 & 0.5 & 2.67 & 1.03 & 0.82 & 0.59 & 0.42 & 0.65 & 2.48 & 0.53 & 1.15 \\
\hline 0.8 & 0.8 & 1.77 & 1.28 & 2.19 & 0.67 & 1.53 & 0.68 & 1.89 & 0.60 & 1.30 \\
\hline 0.8 & 0.7 & 2.00 & 1.27 & 1.75 & 0.61 & 1.49 & 0.57 & 0.87 & 0.49 & 1.41 \\
\hline 0.8 & 0.6 & 2.26 & 1.28 & 0.99 & 0.57 & 0.93 & 0.77 & 1.20 & 0.54 & 1.20 \\
\hline 0.8 & 0.5 & 2.58 & 0.84 & 0.42 & 0.53 & 1.27 & 0.57 & 0.93 & 0.45 & 0.45 \\
\hline 0.7 & 0.7 & 1.95 & 0.91 & 1.40 & 0.49 & 1.48 & 0.55 & 0.98 & 0.46 & 1.26 \\
\hline 0.7 & 0.6 & 2.21 & 1.01 & 1.42 & 0.48 & 0.96 & 0.50 & 1.08 & 0.41 & 1.08 \\
\hline 0.7 & 0.5 & 2.49 & 0.82 & 0.37 & 0.49 & 0.36 & 0.45 & 0.38 & 0.37 & 0.86 \\
\hline 0.6 & 0.6 & 2.16 & 0.72 & 1.14 & 0.36 & 0.98 & 0.45 & 0.41 & 0.32 & 0.41 \\
\hline 0.6 & 0.5 & 2.40 & 0.60 & 0.45 & 0.33 & 0.44 & 0.41 & 1.28 & 0.28 & 1.06 \\
\hline 0.5 & 0.5 & 2.35 & 0.57 & 1.06 & 0.29 & 0.65 & 0.30 & 0.76 & 0.24 & 0.49 \\
\hline \multicolumn{11}{|c|}{ Resolution $=50 \mathrm{k} \mathrm{M}_{\odot}{ }^{-1}$} \\
\hline 1.1 & 1.1 & 1.23 & 3.58 & 6.13 & 1.81 & 8.88 & 1.56 & 3.02 & 1.44 & 3.09 \\
\hline 1.1 & 1.0 & 1.44 & 3.10 & 4.15 & 1.67 & 3.59 & 1.27 & 2.71 & 1.14 & 2.83 \\
\hline 1.1 & 0.9 & 1.65 & 2.20 & 2.87 & 1.32 & 2.66 & 1.13 & 2.94 & 0.99 & 2.36 \\
\hline 1.1 & 0.8 & 1.89 & 1.94 & 1.47 & 1.16 & 1.10 & 1.08 & 2.42 & 0.93 & 2.80 \\
\hline 1.1 & 0.7 & 2.13 & 1.58 & 0.39 & 1.06 & 3.90 & 1.00 & 1.96 & 0.86 & 1.76 \\
\hline 1.1 & 0.6 & 2.42 & 1.52 & 0.33 & 0.88 & 0.51 & 0.94 & 1.89 & 0.82 & 2.19 \\
\hline 1.1 & 0.5 & 2.79 & 1.40 & 0.67 & 0.86 & 0.60 & 0.96 & 1.40 & 0.81 & 1.49 \\
\hline 1.0 & 1.0 & 1.42 & 2.77 & 3.55 & 1.31 & 3.10 & 1.11 & 2.98 & 0.99 & 3.08 \\
\hline 1.0 & 0.9 & 1.62 & 2.16 & 2.67 & 1.06 & 3.01 & 0.94 & 1.87 & 0.81 & 2.73 \\
\hline 1.0 & 0.8 & 1.85 & 1.93 & 1.98 & 1.05 & 1.10 & 0.86 & 1.57 & 0.76 & 1.29 \\
\hline 1.0 & 0.7 & 2.09 & 1.72 & 0.93 & 0.93 & 1.16 & 0.85 & 0.86 & 0.70 & 1.31 \\
\hline 1.0 & 0.6 & 2.37 & 1.28 & 0.50 & 0.78 & 0.56 & 0.79 & 0.88 & 0.66 & 2.01 \\
\hline 1.0 & 0.5 & 2.73 & 1.13 & 0.23 & 0.69 & 0.31 & 0.72 & 1.14 & 0.64 & 1.41 \\
\hline 0.9 & 0.9 & 1.62 & 2.15 & 2.22 & 1.07 & 2.17 & 0.77 & 2.35 & 0.66 & 1.55 \\
\hline 0.9 & 0.8 & 1.84 & 1.59 & 2.08 & 0.83 & 1.92 & 0.69 & 1.55 & 0.58 & 0.85 \\
\hline 0.9 & 0.7 & 2.09 & 1.44 & 0.79 & 0.81 & 1.27 & 0.64 & 0.44 & 0.55 & 0.44 \\
\hline 0.9 & 0.6 & 2.37 & 1.21 & 0.86 & 0.68 & 0.58 & 0.62 & 1.29 & 0.55 & 0.70 \\
\hline 0.9 & 0.5 & 2.73 & 1.00 & 0.29 & 0.59 & 0.18 & 0.63 & 2.51 & 0.50 & 0.37 \\
\hline 0.8 & 0.8 & 1.80 & 1.65 & 1.62 & 0.76 & 1.37 & 0.58 & 1.55 & 0.51 & 1.91 \\
\hline 0.8 & 0.7 & 2.03 & 1.59 & 1.39 & 0.76 & 1.31 & 0.56 & 2.81 & 0.45 & 0.68 \\
\hline 0.8 & 0.6 & 2.30 & 1.21 & 0.56 & 0.60 & 0.40 & 0.51 & 0.62 & 0.42 & 0.62 \\
\hline 0.8 & 0.5 & 2.65 & 0.96 & 0.29 & 0.52 & 0.30 & 0.49 & 0.73 & 0.41 & 0.38 \\
\hline 0.7 & 0.7 & 2.00 & 1.50 & 0.84 & 0.61 & 0.80 & 0.53 & 1.49 & 0.45 & 1.07 \\
\hline 0.7 & 0.6 & 2.29 & 1.10 & 1.04 & 0.53 & 0.80 & 0.45 & 2.94 & 0.34 & 0.32 \\
\hline 0.7 & 0.5 & 2.61 & 0.98 & 0.33 & 0.45 & 0.11 & 0.42 & 0.32 & 0.33 & 0.32 \\
\hline 0.6 & 0.6 & 2.22 & 0.93 & 0.81 & 0.40 & 0.86 & 0.48 & 2.78 & 0.31 & 0.55 \\
\hline 0.6 & 0.5 & 2.52 & 0.74 & 0.48 & 0.41 & 0.36 & 0.36 & 0.32 & 0.25 & 0.32 \\
\hline 0.5 & 0.5 & 2.44 & 0.85 & 0.59 & 0.37 & 0.46 & 0.35 & 1.37 & 0.23 & 0.27 \\
\hline
\end{tabular}


TABLE 2

SUMMARY OF ALL CALCULATED MODELS

\begin{tabular}{|c|c|c|c|c|c|c|c|c|c|c|}
\hline $\begin{array}{c}M_{1} \\
\left(M_{\odot}\right)\end{array}$ & $\begin{array}{c}M_{2} \\
\left(M_{\odot}\right)\end{array}$ & $\begin{array}{c}a_{\text {init }} \\
\left(10^{9} \mathrm{~cm}\right)\end{array}$ & $\begin{array}{c}T_{\max } \\
\left(10^{9} \mathrm{~K}\right)\end{array}$ & $\begin{array}{c}\rho\left(T_{\max }\right) \\
\left(10^{6} \mathrm{~g} \mathrm{~cm}^{-3}\right)\end{array}$ & $\begin{array}{c}T_{\mathrm{s}, \max } \\
\left(10^{9} \mathrm{~K}\right)\end{array}$ & $\begin{array}{c}\rho\left(T_{\mathrm{s}, \max }\right) \\
\left(10^{6} \mathrm{~g} \mathrm{~cm}^{-3}\right)\end{array}$ & $\begin{array}{c}T_{\max , \text { rem }} \\
\left(10^{9} \mathrm{~K}\right)\end{array}$ & $\begin{array}{c}\rho\left(T_{\max , \text { rem }}\right) \\
\left(10^{6} \mathrm{~g} \mathrm{~cm}^{-3}\right)\end{array}$ & $\begin{array}{c}T_{\mathrm{s}, \mathrm{max}, \text { rem }} \\
\left(10^{9} \mathrm{~K}\right)\end{array}$ & $\begin{array}{c}\rho\left(T_{\mathrm{s}, \text { max }, \text { rem }}\right) \\
\left(10^{6} \mathrm{~g} \mathrm{~cm}^{-3}\right)\end{array}$ \\
\hline \multicolumn{11}{|c|}{ Resolution $=100 k \mathrm{M}_{\odot}{ }^{-1}$} \\
\hline 1.1 & 1.1 & 1.25 & 3.64 & 5.51 & 1.79 & 3.38 & 1.39 & 2.82 & 1.25 & 2.88 \\
\hline 1.1 & 1.0 & 1.45 & 3.02 & 6.71 & 1.67 & 3.05 & 1.23 & 2.67 & 1.10 & 3.11 \\
\hline 1.1 & 0.9 & 1.67 & 2.54 & 4.13 & 1.46 & 4.31 & 1.17 & 2.74 & 1.01 & 1.73 \\
\hline 1.1 & 0.8 & 1.86 & 2.21 & 1.92 & 1.28 & 1.67 & 1.08 & 2.12 & 0.96 & 2.96 \\
\hline 1.1 & 0.7 & 2.11 & 1.82 & 1.10 & 1.09 & 0.90 & 0.98 & 2.47 & 0.90 & 1.51 \\
\hline 1.1 & 0.6 & 2.39 & 1.56 & 0.42 & 0.91 & 0.65 & 0.95 & 1.82 & 0.82 & 1.24 \\
\hline 1.1 & 0.5 & 2.72 & 1.42 & 1.91 & 0.77 & 0.88 & 0.92 & 0.67 & 0.81 & 0.83 \\
\hline 1.0 & 1.0 & 1.43 & 3.08 & 4.80 & 1.57 & 4.17 & 0.98 & 3.05 & 0.86 & 2.57 \\
\hline 1.0 & 0.9 & 1.64 & 2.10 & 3.32 & 1.17 & 2.14 & 0.93 & 1.15 & 0.80 & 1.70 \\
\hline 1.0 & 0.8 & 1.87 & 1.99 & 2.49 & 1.17 & 1.07 & 0.84 & 0.73 & 0.74 & 0.97 \\
\hline 1.0 & 0.7 & 2.13 & 1.64 & 1.05 & 0.98 & 0.62 & 0.81 & 0.38 & 0.68 & 0.45 \\
\hline 1.0 & 0.6 & 2.40 & 1.45 & 1.23 & 0.89 & 0.76 & 0.77 & 0.98 & 0.66 & 1.16 \\
\hline 1.0 & 0.5 & 2.75 & 1.30 & 0.18 & 0.70 & 0.32 & 0.75 & 0.90 & 0.62 & 0.78 \\
\hline 0.9 & 0.9 & 1.64 & 2.17 & 3.21 & 0.95 & 2.34 & 0.78 & 2.92 & 0.66 & 2.09 \\
\hline 0.9 & 0.8 & 1.86 & 1.96 & 1.24 & 1.07 & 1.05 & 0.71 & 1.26 & 0.60 & 0.97 \\
\hline 0.9 & 0.7 & 2.08 & 1.48 & 1.51 & 0.84 & 0.56 & 0.66 & 0.40 & 0.59 & 0.38 \\
\hline 0.9 & 0.6 & 2.35 & 1.27 & 0.67 & 0.73 & 0.39 & 0.65 & 0.56 & 0.58 & 0.72 \\
\hline 0.9 & 0.5 & 2.68 & 1.07 & 0.31 & 0.61 & 0.18 & 0.59 & 0.48 & 0.50 & 0.50 \\
\hline 0.8 & 0.8 & 1.83 & 1.65 & 2.20 & 0.83 & 3.35 & 0.59 & 2.92 & 0.54 & 1.31 \\
\hline 0.8 & 0.7 & 2.07 & 1.51 & 0.89 & 0.81 & 0.82 & 0.58 & 0.79 & 0.46 & 0.97 \\
\hline 0.8 & 0.6 & 2.35 & 1.27 & 0.56 & 0.63 & 0.43 & 0.57 & 0.43 & 0.45 & 0.37 \\
\hline 0.8 & 0.5 & 2.69 & 1.19 & 0.37 & 0.59 & 0.19 & 0.50 & 2.74 & 0.40 & 0.51 \\
\hline 0.7 & 0.7 & 2.01 & 1.44 & 1.12 & 0.80 & 1.10 & 0.49 & 0.86 & 0.42 & 0.79 \\
\hline 0.7 & 0.6 & 2.27 & 1.12 & 0.45 & 0.63 & 0.39 & 0.47 & 0.60 & 0.35 & 0.46 \\
\hline 0.7 & 0.5 & 2.60 & 0.84 & 0.45 & 0.49 & 0.30 & 0.46 & 0.35 & 0.34 & 0.32 \\
\hline 0.6 & 0.6 & 2.25 & 1.13 & 0.76 & 0.58 & 0.67 & 0.49 & 2.61 & 0.28 & 0.43 \\
\hline 0.6 & 0.5 & 2.55 & 0.81 & 0.44 & 0.44 & 0.30 & 0.32 & 2.64 & 0.25 & 0.35 \\
\hline 0.5 & 0.5 & 2.47 & 0.85 & 0.52 & 0.42 & 0.47 & 0.40 & 1.89 & 0.22 & 0.32 \\
\hline \multicolumn{11}{|c|}{ Resolution $=500 k \mathrm{M}_{\odot}{ }^{-1}$} \\
\hline 1.1 & 1.1 & 1.25 & 3.91 & 5.84 & 2.28 & 5.27 & 1.18 & 3.10 & 0.99 & 2.90 \\
\hline 1.1 & 1.0 & 1.46 & 3.50 & 3.81 & 2.52 & 3.87 & 1.18 & 2.41 & 1.02 & 1.63 \\
\hline 1.1 & 0.9 & 1.67 & 2.58 & 4.24 & 1.74 & 3.96 & 1.10 & 1.48 & 1.01 & 1.11 \\
\hline 1.1 & 0.8 & 1.91 & 2.19 & 2.23 & 1.43 & 1.67 & 1.04 & 0.54 & 0.94 & 1.01 \\
\hline 1.1 & 0.7 & 2.16 & 1.66 & 1.50 & 1.18 & 0.69 & 0.96 & 0.99 & 0.85 & 0.91 \\
\hline 1.1 & 0.6 & 2.45 & 1.68 & 0.70 & 1.00 & 0.57 & 0.95 & 0.74 & 0.82 & 0.89 \\
\hline 1.1 & 0.5 & 2.81 & 1.70 & 0.76 & 0.82 & 0.22 & 0.90 & 0.63 & 0.78 & 0.61 \\
\hline 1.0 & 1.0 & 1.43 & 3.50 & 4.27 & 1.61 & 5.76 & 0.92 & 2.97 & 0.80 & 1.82 \\
\hline 1.0 & 0.9 & 1.64 & 2.95 & 3.78 & 1.98 & 1.81 & 0.90 & 1.89 & 0.78 & 1.77 \\
\hline 1.0 & 0.8 & 1.87 & 2.26 & 1.32 & 1.54 & 1.32 & 0.87 & 0.91 & 0.77 & 0.73 \\
\hline 1.0 & 0.7 & 2.11 & 1.87 & 0.93 & 1.19 & 1.13 & 0.84 & 1.06 & 0.73 & 0.33 \\
\hline 1.0 & 0.6 & 2.40 & 1.31 & 0.65 & 0.69 & 0.31 & 0.79 & 0.72 & 0.66 & 1.11 \\
\hline 1.0 & 0.5 & 2.74 & 1.49 & 0.58 & 0.78 & 0.15 & 0.78 & 0.74 & 0.63 & 0.51 \\
\hline 0.9 & 0.9 & 1.64 & 2.77 & 3.84 & 1.36 & 2.94 & 0.79 & 2.66 & 0.56 & 1.19 \\
\hline 0.9 & 0.8 & 1.86 & 2.17 & 1.26 & 1.40 & 0.77 & 0.64 & 0.87 & 0.57 & 1.03 \\
\hline 0.9 & 0.7 & 2.11 & 1.72 & 0.50 & 1.05 & 0.85 & 0.64 & 0.38 & 0.57 & 0.40 \\
\hline 0.9 & 0.6 & 2.40 & 1.30 & 0.43 & 0.88 & 0.36 & 0.63 & 0.51 & 0.52 & 0.46 \\
\hline 0.9 & 0.5 & 2.75 & 1.28 & 0.24 & 0.70 & 0.20 & 0.59 & 0.60 & 0.50 & 0.36 \\
\hline 0.8 & 0.8 & 1.82 & 2.16 & 2.08 & 1.15 & 1.71 & 0.57 & 1.86 & 0.47 & 0.44 \\
\hline 0.8 & 0.7 & 2.06 & 1.74 & 0.80 & 0.96 & 0.73 & 0.50 & 0.82 & 0.43 & 0.53 \\
\hline 0.8 & 0.6 & 2.34 & 1.38 & 0.75 & 0.81 & 0.46 & 0.50 & 0.54 & 0.44 & 0.24 \\
\hline 0.8 & 0.5 & 2.67 & 1.04 & 0.33 & 0.54 & 0.11 & 0.50 & 0.49 & 0.41 & 0.17 \\
\hline 0.7 & 0.7 & 2.05 & 1.63 & 1.38 & 0.73 & 0.93 & 0.51 & 1.71 & 0.37 & 0.59 \\
\hline 0.7 & 0.6 & 2.31 & 1.11 & 0.55 & 0.57 & 0.52 & 0.48 & 3.13 & 0.33 & 0.22 \\
\hline 0.7 & 0.5 & 2.65 & 1.07 & 0.25 & 0.49 & 0.19 & 0.44 & 2.57 & 0.34 & 0.15 \\
\hline 0.6 & 0.6 & 2.24 & 1.45 & 1.21 & 0.79 & 0.64 & 0.50 & 1.90 & 0.27 & 0.49 \\
\hline 0.6 & 0.5 & 2.55 & 0.93 & 0.52 & 0.49 & 0.16 & 0.40 & 1.77 & 0.25 & 0.10 \\
\hline 0.5 & 0.5 & 2.47 & 1.09 & 0.52 & 0.56 & 0.29 & 0.41 & 1.50 & 0.21 & 0.38 \\
\hline
\end{tabular}

